\title{
Suppressing reflexive behaviour Saccadic eye movements in musicians and non-musicians
}

\author{
${ }^{1}$ Wilfried Gruhn, ${ }^{1}$ Friederike Litt, ${ }^{1}$ Annette Scherer, ${ }^{1}$ Till Schumann, ${ }^{1}$ Eva Maria \\ Weiß, ${ }^{2}$ Christine Gebhardt
}

1) Music Education Department; University of Music Freiburg, Germany

2) Blick Labor, Brain Research Unit, University of Freiburg, Germany

\begin{abstract}
Musicians who practice from notation and sight read every day can be said to perform a special eye training and could therefore possibly be distinguished from non-musicians. If a difference between both groups could be demonstrated it would be interesting to know whether this difference remains stable over the entire life span. In a cross-sectional study we tested 115 subjects of three age groups ( 36 pupils $M=11.5$ years, 41 university students $M=23.1$ years, 38 adults 55.6 years) and varying degrees of musical training (57 were musicians) with respect to their saccadic eye movements (overlap and anti gap paradigm). An infra-red beam helmet (Express Eye) was used to collect data for the reaction time of pro and anti saccades, mean distribution, percentage of express saccades, correction time, and percentage of directional errors. Data were analyzed separately for each age group and served as factors for fixation and voluntary control. Data from measures of general intelligence (Raven's Advanced Progressive Matrices), music aptitude (Gordon's Advanced Measures of Music Audiation), handedness, and sight reading were used as covariates. The data exhibit an advantage for musicians regarding those parameters that involve involuntary control and fixation. But in general there is no evidence to show that music aptitude and saccadic behavior interact.
\end{abstract}




\section{Introduction}

Along with the debate on the cognitive transfer effects of music, several investigations have focused on the relation between music and intelligence (Winner \& Hetland 2000; Bastian 2000; Petsche 2002; Gruhn, Galley \& Kluth 2003). According to Gardner's theory of multiple intelligences music aptitude or talent can be seen as one intelligence amongst others (Gardner 1985 ; 1999) or it can be interpreted as the performance of a special intelligence on a subordinate level of general intelligence when $\mathrm{g}$ factor is positioned at the top and specific abilities at the bottom of a hierarchical structure of the intelligence pyramid.

Based on this assumption music education research became involved in the investigation of saccadic eye movement because oculo-motor tasks can be related to mental speed and may reflect mental abilities and disabilities. Saccades are rapid eye movements between phases of fixation which are used in dynamic vision to focus on particular objects. In natural visual perception human eyes execute three to five rapid movements (saccades) per second at various time ranges: fast saccades occur within 135 to 180 milliseconds; saccadic reactions within 80 to 135 milliseconds are referred to as express-saccades which cannot be seen as actual reactions instead of anticipations. Below this threshold we enter the realm of mere reflexes.

With regard to the very complex nature of vision control and eye movement, it has been questioned whether mental speed measured by saccadic reaction time possibly interacts with music achievement. A significant interaction of eye movement and musical activities has been found in one study (Gruhn, Galley, \& Kluth 2003) in which subjects followed a gradually accelerating running or jumping spot (from left to right etc., or up and down) with their eyes. In this task, the reaction time (time span between the appearance of the stimulus and the beginning of the eye movement following the stimulus), the anticipation of the turning of the stimulus, and the omissions present a valid indicator for mental speed (speed of mental processing) which significantly correlates with cognitive intelligence (Saring \& v. Cramon 1980; Galley 1999).

In previous experiments, two paradigms for saccadic eye movement, namely the overlap and anti-gap task (see Figure 1) have been investigated more intensively. It was shown that unstable fixation and weak voluntary control may function as an indicator for dyslexia (Fischer 1999; Biscaldi et. al. 2000). Furthermore, effects of Alzheimer and Huntington Disease (Currie et al. 1991), and of schizophrenia (Rosse et al. 1993; Sereno et al. 1995) on saccadic eye movement have been investigated, and, oculo-motor anomalies have been reported for children with Attention Deficit and Hyperactivity Disorder, (Mokler, 2002). In music saccadic eye movements have been investigated while subjects (trained musicians and amateurs) were sight reading musical scores (Kinsler \& Carpenter 1995).

According to these studies, it appears likely that a direct connection between attention and saccadic eye movement exists. Voluntarily controlled eye movement and fixation follow a highly complex mental process (Tatler \& Wade 2003) involving many brain areas. Most of the parameters of the eye movement applied to our study //unclear// reveal a significant age effect. Visual acuity has been fully developed by the age of 8 to 9 years (Fischer 1999). However, control over saccades by way of fixation and voluntary eye movement by suppression of reflexive behaviour can still improve until 20 years of age followed by a moderate, but accelerating decline with advancing age (Fischer 1999; 2003).

Furthermore, all modalities of attention have an impact on the oculo-motor system (Kimmig 1986). The overlap task constitutes an important factor for attention because the fixation ( $F$ factor measured by reaction time in pro-saccades) which is characterized by a voluntary suppression of saccades and the rate of express saccades determine processes in the frontal lobe where attention is generated. On the other hand, the mean reaction time, the correction time and the number of corrections for directional errors in anti-gap tasks indicate voluntary eye control (V factor). Both factors - concentration and stability as well as voluntary control of reactions - 
are absolutely vital for all kinds of music performance. Therefore, it seems reasonable to observe and investigate these factors in musicians and non-musicians. The main goal of the experiments was to determine whether the two factors of eye movement interact with musical activities and music aptitude. Questions to be answered were:

- Do musically trained subjects differ from non-musicians regarding their saccadic reaction time in overlap and anti-saccades tasks, number of direction errors and the percentage and time for corrections?

- Does musical practice have a positive effect on the stability of fixations and the voluntary control of saccadic eye movement?

- And if this can be confirmed, does it mean that there is a physiological transfer effect of music on brain activities in other domains such as visual control?

\section{Experiment 1}

\section{Subjects}

Samples of children from three age groups representing different stages of development were observed. Altogether, 115 subjects volunteered for the study:

- 36 high school students (mean age $=11,5$ years, 18 male, 18 female), among those 21 musicians who had ongoing instrumental training for at least four years $(10=48 \%$ keyboard instruments; $11=52 \%$ string or wind instruments) and 15 students without any musical training;

- 41 university students (mean age $=23,1$ years, $16 \mathrm{~m}, 25 \mathrm{f}$ ) of whom 21 were music majors of the Music Academy Freiburg $(8=28 \%$ piano; $13=62 \%$ string or wind instruments; however, all played the piano as their second instrument) and 20 non music university students;

- a group of 38 adults (mean age $=55,6$ years, $22 \mathrm{~m}, 16 \mathrm{f}$ ) consisting of 15 professional musicians ( $9=60 \%$ piano; $6=40 \%$ string and wind instruments) and 23 adult patients of the Blick Labor Freiburg with no special musical training.

Subjects were predominantly right handed (87.0\% right, $7.6 \%$ left, 5.4\% mixed).

\section{Method}

To measure $\mathrm{F}$ and $\mathrm{V}$ factors across the age range, two paradigms were applied.

(1) The overlap paradigm

A central fixation point was presented to the subjects to focus their visual fixation. After a few seconds a peripheral stimulus was added simultaneously as target stimulus (the "overlap"). The subjects were asked to immediately move the eyes toward the target stimulus. The target stimulus appeared 50 times on either side, right and left, at an angle of $\pm 4^{\circ}$ in randomised order so that a total of 100 trials were performed (see Figure 1).

(2) The gap paradigm

In this paradigm, a pause of $200 \mathrm{~ms}$ (the "gap") occurred between the central and the target stimulus so that the central stimulus disappeared before the target stimulus was presented. The target stimulus appeared 50 times on either side, right and left, with an angle of $\pm 4^{\circ}$ in randomised order resulting in a total of 100 trials (Figure 1).

Within this condition, two different tasks had to be performed, a pro-saccade and an antisaccade task. In the pro-saccade task, the eyes followed the target stimulus, whereas in the antisaccade task subjects were asked to immediately move their eyes into the opposite direction, calling for voluntary control (see Figure 2). 


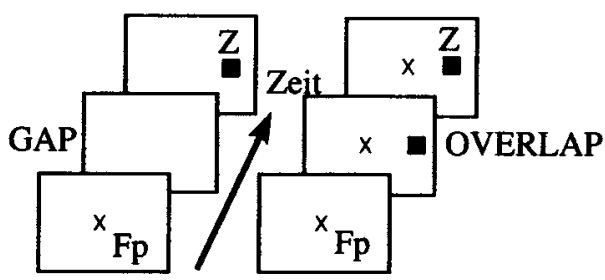

\section{Figure 1}

Model of the gap and overlap paradigm. The graph shows a screen in sequential order (from front to back) with fixation point (Fp) and target stimulus (Z). (Fischer 1999, p. 146).

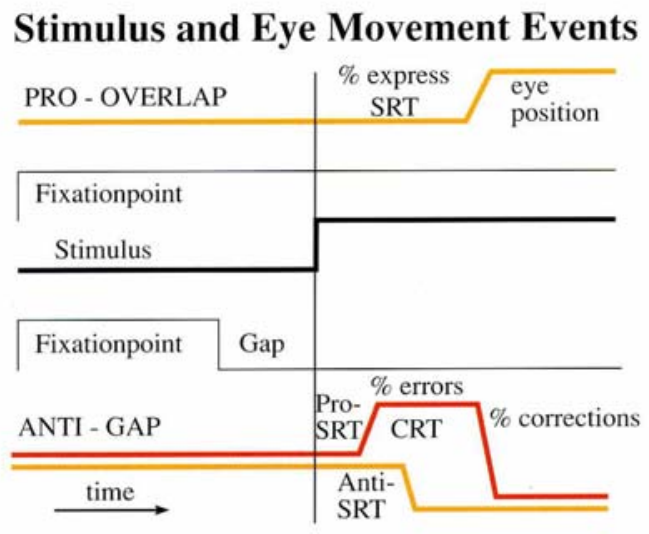

\section{Figure 2}

General design of the pro-overlap and anti-gap paradigm. The middle line represents the stimulus. The upward direction refers to a jump to the right, downwards refers to the left. The distance between the stimulus and the delayed eye movement indicates the saccadic reaction time (SRT). The bottom line shows a directional error which is corrected immediately. The distance between the two opposite movements is referred to as correction reaction time (CRT).

(C) Blick Labor Freiburg).

Mean reaction time, distribution of reaction time, number of express saccades, rate of directional errors, and the reaction time for corrections within the anti-gap task were recorded during the series of 100 trials each (pro overlap and anti gap). The average reaction time, i.e. the distance between stimulus perception and motor reaction, was about $200 \mathrm{~ms}$. Extremely short reactions within $80-135 \mathrm{~ms}$ were labeled express saccades and function as a strong indicator for fixation control.

Measurements were taken individually using a helmet with an integrated infra-red diode and two photo sensors directed toward both edges of the cornea. A small laser pointer on top of the helmet projected a stimulus on a screen in front of the subject. While moving their eyes the infrared beam is reflected depending on the shift of the eyes. For each subject, the sensors had to be calibrated. Subjects were placed in a quiet and darkened room and performed both tasks after verbal instruction and a brief training phase. Additionally, all subjects were controlled with respect to intelligence, music aptitude, and handedness. Additionally, a sight reading test was carried out mainly with the intention to control the subjects' ability to perform as well as the validity of their musical practice. Therefore, every subject performed the following tests in a separate session: Raven's Advanced Progressive Matrices (APM, group testing) for intelligence, 
Gordon's Advanced Measures of Music Audiation, (AMMA, group testing) for music aptitude, the Edinburgh Inventory (Oldfield 1971) for handedness (questionnaire), and the musicians played an Etude by M. Wright arranged for their major instrument which was recorded on a mini disc and adjudicated on a six point rating scale. The collected data were statistically analysed using Spearman correlation ranks, Mann-Whitney U-Test, and a multivariate analysis with repeated measures on all variables of the tests.

\section{Results}

As expected, reaction times of all groups showed two distinct peaks, one at about $120 \mathrm{~ms}(=$ express saccades) and the other at about 140-150 ms. However, this was most prominent in the anti-gap task. Fixation and voluntary control as measured by the reaction time of pro-overlap and anti-gap tasks showed a clear age effect. Up to 20 years of age the reaction time improves (i.e. diminishes) in both groups, musicians and non-musicians, but deteriorates (i.e. increases) with age. In general, there are no striking differences for many parameters within age groups. However, it is important to review those parameters which exhibit differential effects. High school musicians differed significantly from their non-musician peers regarding the express saccades $(\mathrm{p}=.039)$, whereas university students only showed a slight tendency with regard to the amount of errors (anti-gap errors $p=.068$ ) [direction of effect unclear!! more errors]. However, adults differ significantly with regard to their anti-gap reaction time $(p=.055)$ and the rate of their directional error corrections $(\mathrm{p}=.016)$. For these parameters, musicians exhibited higher rates of corrections and shorter reaction times.

A multivariate analysis of variance for all variables (pro-overlap: mean reaction time, distribution, rate of express saccades; anti-gap: mean reaction time for pro- and anti-saccades, number of directional errors, mean reaction time for corrections, rate of corrections) with musical activity and age as independent factors demonstrated that only age has an highly significant $(\mathrm{F}=4.168 ; \mathrm{p}<.01)$ impact on the mean of all variables whereas musical activity only shows a slight and non-significant tendency $(\mathrm{F}=1.694 ; \mathrm{p}=.058)$.

Although there was no overall significant difference between musicians and non-musicians which could be attributed to oculo-motor advantages caused by musical training, it was obvious that musicians tended to exceed their non-musician peers with regard to those factors that call for voluntary control and fixation stability. This is reflected by figures 3.1 and 3.2, which show reaction times for saccades and correction times for errors in the anti-saccades in the antisaccade task. Although the difference is not statistically significant, a slight tendency towards shorter reaction times appears especially in the musicians' group of experiment 2.

Another clear distinction between musicians and non-musicians was revealed by conspicuous divergences in the oculo-motor tasks. Non-musicians produced significantly larger standard deviations than non-musicians $(\mathrm{p}=.007)$ in their voluntary control data in anti-gap tasks, where frontal brain activities are involved. This indicates that musicians have a stronger voluntary control at their disposal which enables them to suppress reflex reactions. On the other hand, neither music aptitude, nor handedness, nor general intelligence showed any effect on any one of the oculo-motor variables. However, if we extract the best musicians (identified by the scores in the sight reading and music aptitude tests) from the total sample, these subjects were always found to produce above average results compared to the non-musicians. 


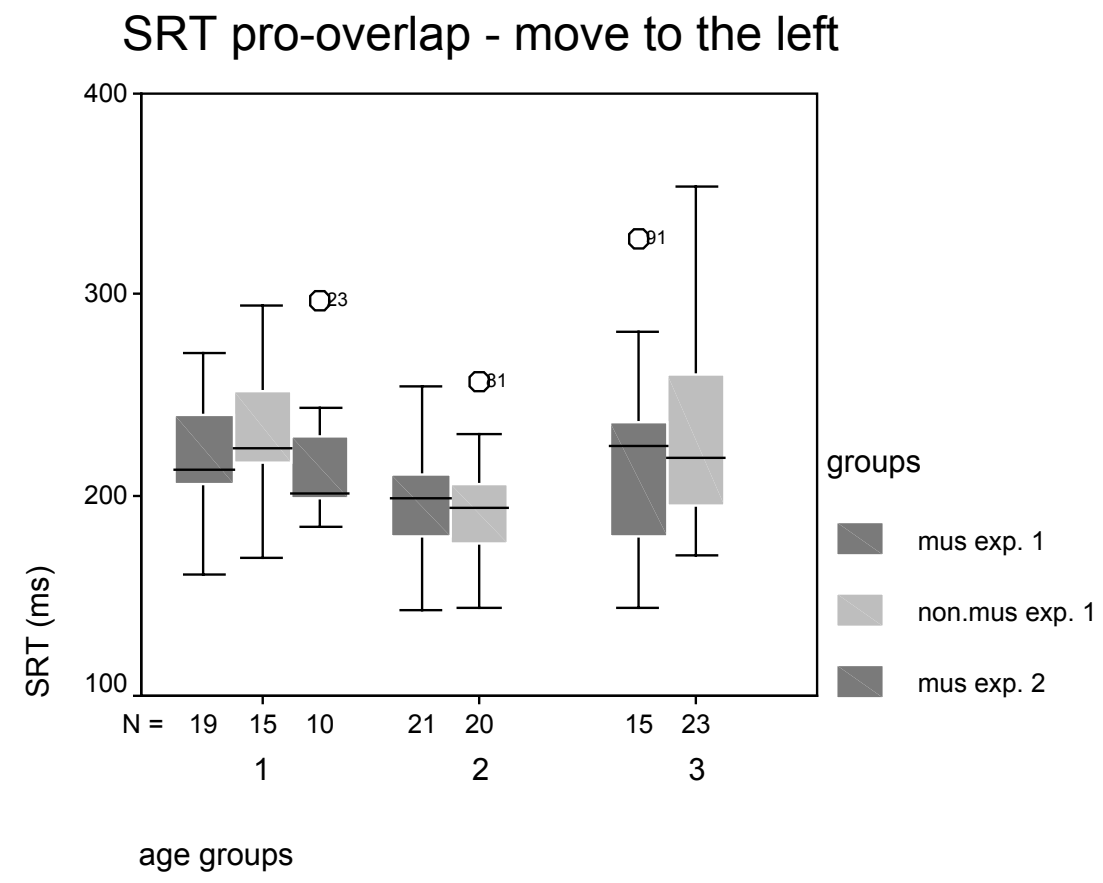

Figure 3.1

Boxplot of saccadic reaction times (SRT) in the pro-overlap task for musicians and non-musicians for all age groups when the stimulus moves to the left.

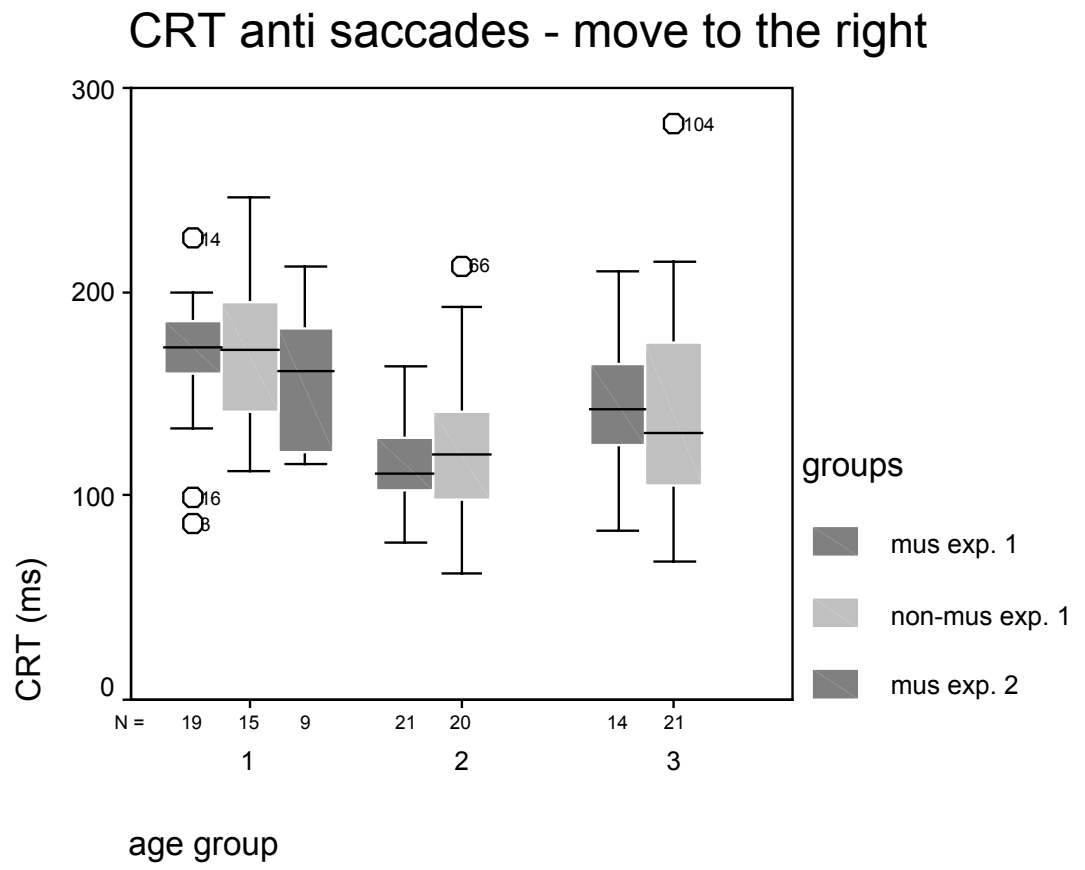

\section{Figure 3.2}

Boxplot of correction reaction time (CRT) for anti-saccades in musicians and non-musicians for all age groups when the stimulus moves to the right. 


\section{Experiment 2: Control condition}

Contrary to our expectations, the musically active high school students did not exhibit valid differences compared with their non-musician peers. This could be due to the fact that they only identified themselves as "musicians" because they had played an instrument for at least four years. However, their sight-reading test showed rather poor results suggesting that their selfidentification could not be taken as a valid criterion for musical competence. Moreover, there was neither a performance assessment nor a quantitative measure of experience (e.g., amount of practice time). This group might therefore not really differ from the self-proclaimed nonmusicians with regard to their relevant eye training. Therefore, in a second experiment a group of verified musically experienced students was tested and compared with the non-musicians from the first experiment.

\section{Subjects}

Ten instrumentalists (mean age $=12.3$ years; $5 \mathrm{~m}, 5 \mathrm{f}$ ) from a local high school were designated by their teachers as experienced sight readers and talented instrumental students volunteered in the experiment. Now, this group of 3 pianists and 7 string and wind instrumentalists was significantly differentiated from non-musicians by their daily practice time $(p=.025)$ and the scores of their sight reading test $(\mathrm{p}<.01)$

\section{Method}

Procedures and measurements were the same as in the first experiment. All subjects performed the same oculo-motor tasks.

\section{Results}

This group of musically experienced students differed significantly from non-musicians (taken from the first experiment) regarding their reaction times for correct anti-saccades in the anti-gap condition which is the most advanced task and calls for voluntary inhibition of reflex reactions (see Figure 4). Generally, the musically experienced subjects from this group also exhibited faster reaction times in the overlap condition and a moderately higher percentage of corrections in the anti-saccades task, but these results were not statistically significant. Still, shorter reaction times and fewer errors would be consistent with a stronger voluntary control that musicians possess who read music while practicing their instruments and, therefore, undergo a specialized training. This was reflected by better results in eye movement control. 


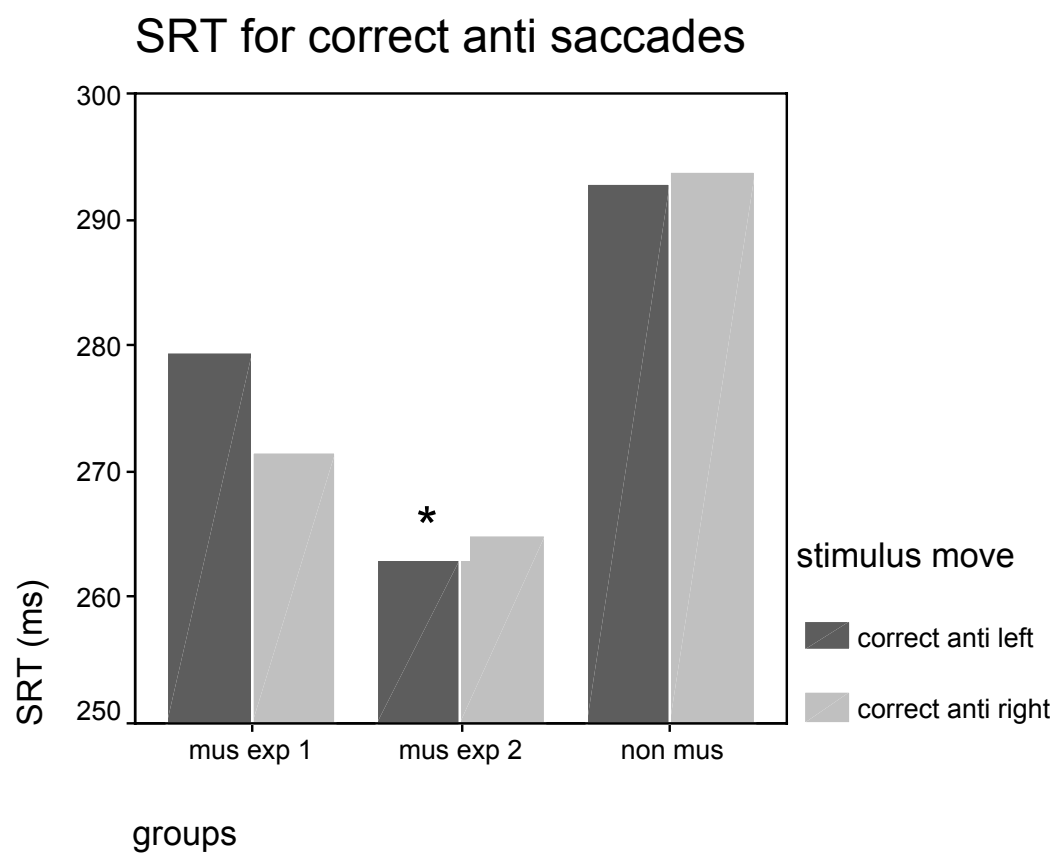

\section{Figure 4}

Reaction times of musicians and non-musicians for correct anti-saccades of left (left column) and right (right column) eye movements. Only times of right eye movements differ significantly $(p=.016)$ in musicians of experiment 2 from non-musicians.

\section{Discussion and conclusions}

Although the data of this study do not present a final confirmation that musical practice has an impact on the performance of saccadic eye movements in general, a clear tendency became apparent: subjects who trained in music did better in those tasks in which frontal areas of the brain are involved and spontaneous reactions are essential. In general, musicians performed at higher speeds, their reaction times were shorter, they produced more express saccades, and they were more successful in their correction of directional errors. They are also significantly more successful in saccadic suppression. Furthermore, it could be shown that even the best musicians in our study had a stronger voluntary eye control and were more stable in their fixations. This is not surprising and probably due to their daily music training which calls for high concentration, controlled eye fixation, focussed attention and voluntary control of all fine-motor movement involved in music performance.

The lack of statistically significant differences between musicians and non-musicians across all parameters was due to a very broad distribution within each group of this small sample. However, we have to emphasize that especially those parameters which refer to voluntary control and require saccadic suppression clearly indicated a significant difference between musicians and non-musicians. The neural mechanisms of saccadic suppression have recently been described (Thiele et al. 2002; Thilo et al. 2004) for visual perception, but they also apply to activities that require eye-control. Therefore, saccadic suppression is not an arbitrary factor in voluntary control.

This confirms findings from an earlier study (Gruhn, Galley, \& Kluth 2002) where oculomotor data were collected from a continuously accelerated running and jumping spot paradigm 
and interpreted as an indicator of mental speed. Only young children who were musically engaged in manifold ways had participated in this previous study. Their data were referenced with those of a large population of peers without any musical training. It was demonstrated that all subjects with musical training exceeded their peers significantly in mental speed.

It is also evident that stronger $\mathrm{F}$ and $\mathrm{V}$ factors in musicians were reflected by fixation data such as mean reaction time of pro-saccades and rate of express saccades and voluntary control such as mean reaction time of anti-saccades, correction time, and rate of direction errors. Additionally, it is likely that musicians perform at higher levels of mental speed.

We could speculate that that mental speed can be seen as a very general measure for cognitive potential which is a necessary prerequisite for the development of specific aptitudes. Alternatively, the results could support the idea that general factors like power of concentration, reaction time, voluntary control, and control of gross and fine motions are enhanced by music practice, speaking in favour of the theory of transfer effects of music training.

Neither this study nor former experiments can provide a causal link between music aptitude and achievement on the one side and cognitive factors like mental speed on the other. The data of our present study make it more likely to assume that musical and cognitive abilities develop independently. Both faculties seem to rely on general abilities that share mental speed, fixation, and voluntary control factors. Those factors are reflected in the efficiency of eye movement. However, this study did not support the conclusion that musicians are generally superior with regard to their oculo-motor capacities. 


\section{References}

Bastian, H.G. (2000). Musik(erziehung) und ihre Wirkung. Mainz: Schott.

Biscaldi, M., Fischer, B., \& Hartnegg, K. (2000). Voluntary saccadic control in dyslexia. Perception 29, $509-521$.

Currie, J., Ramsden, B., McArthur, C., \& Maruff, P. (1991). Validation of a clinical saccadic eye movement test in the assessment of dementia. Archives of Neurology 48, 644-648.

Fischer, B. (1999). Blick-Punkte. Neurobiologische Prinzipien des Sehens und der Blicksteuerung. Bern: Huber.

Fischer, B. (2003). Hören, Sehen, Blicken, Zählen. Teilleistungen und ihre Störungen. Bern: Huber.

Galley, N. (1999). Fixation durations and saccadic latencies as indicators of mental speed. In I. Mervielde, I. J. Deary et al. (eds.). Personality Psychology, vol. 7 (pp. 221- 234). Tilburg: University Press.

Gardner, H. (1999). Intelligence reframed. New York: Basic Books

Gardner, H. (1985). Frames of mind. The theory of multiple intelligences. New York: Basic Books.

Gruhn, W., Galley, N., \& Kluth, Ch. (2003). Do mental speed and musical abilities interact? Annals of the New York Academy of Sciences, 999, 485 - 496.

Kimmig, H. (1986). Express-Sakkaden beim Menschen: Die Rolle der Aufmerksamkeit in der Vorbereitungsphase zielgerichteter Blicksprünge. Diss. med. Universität Freiburg 1990.

Kinsler, V.\& R. H. Carpenter (1995). Saccadic eye movements while reading music. Vision Research 35 (10), 1447 - 1458.

Mokler, A.V. (2002). Okulomotorik bei aufmerksamkeitsgestörte, hyperaktiven Kindern. Diss. Universität Freiburg. www.freidok.uni-freiburg.de/volltexte/326.

Oldfield, R. C. (1971). The assessment and the analysis of handedness: the Edinburgh Inventory. Neuropsychologia, 9, $97-113$.

Petsche, H. (2002). Gehirn - Musik - Intelligenz. Verhandlungen der Deutschen Gesellschaft für Pathologie, 86, 131 - 137.

Rosse, R. B., Schwartz, B. L., Kim, S. Y., \& Deutsch, S. I. (1993). Correlation with antisaccadic and Wisconsin Card Sorting Test in schizophrenia. American Journal of Psychiatry 150, 333 -335 .

Saring, W., \& v. Cramon, D. (1980). Is there an interaction between cognitive ability and lateral eye movements? Neuropsychologia 18 (4-5), $591-596$.

Tatler, B. W., \& Wade, N. J. (2003). On nystagmus, saccades, and fixations. Perception 32 (2), $167-184$.

Sereno, A. B. \& Holzman, P. S. (1995). Antisaccades and smooth pursuit eye movements in schizophrenia. Biological Psychiatry 37, $394-401$.

Thiele, A., Henning, P., Kubischik, M., \& Hoffmann, K.-P. (2002). Neural mechanisms of saccadic suppression. Science 295 (5564), 2460 - 2462.

Thilo, K.V., Santoro, L., Walsh, V., \& Blakemore, C. (2004). The site of saccadic suppression. Nature Neuroscience, 7(1), 13 - 14.

Winner, E., \& Hetland, L. (2000). The arts in education: Evaluating the evidence for a causal link. The Journal of Aesthetic Education, 34(3-4), 3 -10. 